\title{
A pharmacodynamic analysis of factors affecting recovery from anesthesia with propofol-remifentanil target controlled infusion
}

\author{
Bon-nyeo $\mathrm{KOO}^{1,2}$, Jeong-rim LEE ${ }^{1,2}$, Gyu-jeong $\mathrm{NOH}^{3}$, Jae-hoon $\mathrm{LEE}^{1,2}$, Young-ran $\mathrm{KANG}^{1}$, Dong-woo HAN ${ }^{1,2, *}$ \\ ${ }^{1}$ Department of Anesthesiology and Pain Medicine and ${ }^{2}$ Anesthesia and Pain Research Institute, Yonsei University College of Medi- \\ cine, Seoul 120-752, Korea; ${ }^{3}$ Department of Clinical Pharmacology and Therapeutics/Anesthesiology and Pain Medicine, Asan \\ Medical Centre University of Ulsan College of Medicine, Seoul 138-736, Korea
}

\begin{abstract}
Aim: To examine individual patient's demographic parameters and clinical variables related to return of consciousness (ROC) and the pharmacodynamic relationship between propofol effect-site concentration $\left(C_{e}\right)$ and ROC from propofol-remifentanil anesthesia. Methods: Ninety-four patients received propofol-remifentanil anesthesia using the effect-site target-controlled infusion (TCl) system. All clinical events were noted, and variables possibly related to propofol $C_{e}$ at ROC were examined using linear correlation analyses. Pharmacodynamic modeling incorporating covariates was performed using NONMEM (Nonlinear Mixed Effects Modeling) VII software. Results: The $C_{\mathrm{e}}$ values of propofol at loss of consciousness (LOC) and ROC were $4.4 \pm 1.1 \mu \mathrm{g} / \mathrm{mL}$ and $1.1 \pm 0.3 \mu \mathrm{g} / \mathrm{mL}$, respectively. Age was negatively correlated with propofol $C_{\mathrm{e}}$ at ROC $(r=-0.48, P<0.01)$. Including age as a covariate in $C_{\text {e50 }}$ (the effect-site concentration associated with $50 \%$ probability of return of consciousness) and $\lambda$ (the steepness of the concentration-versus-response relationship) significantly improved the performance of the basic model based on the likelihood ratio test, with a significant decrease in the minimum value of the objective function. The $C_{\mathrm{e} 50}$ in $25-, 50$-, and 75 -year-old patients was predicted to be $1.38,1.06$, and $0.74 \mu \mathrm{g} / \mathrm{mL}$, respectively. The $\lambda$ in 25-, 50-, and 75-year-old patients was predicted to be $12.23,8.70$, and 5.18, respectively.

Conclusion: Age significantly affects the relationship between propofol $C_{\mathrm{e}}$ and ROC, and pharmacodynamic modeling including age could lead to better predictions of ROC during emergence from propofol-remifentanil anesthesia.
\end{abstract}

Keywords: anesthesia; effect-site concentration; propofol; pharmacodynamic modeling; return of consciousness; target-controlled infusion

Acta Pharmacologica Sinica (2012) 33: 1080-1084; doi: 10.1038/aps.2012.85; published online 30 Jul 2012

\section{Introduction}

Anesthesiologists are concerned not only about inducing rapid and safe anesthesia, but also about achieving a comfortable and precise return of consciousness (ROC) after surgery ${ }^{[1]}$. To date, studies have focused mainly on individual effects of anesthesia induction. The ability to predict the individual propofol effect-site concentration $\left(C_{\mathrm{e}}\right)$ for ROC would allow the dose of propofol to be adjusted to achieve an adequate ROC. It would also reduce the anesthesiologists workload, save time and resources, and allow for safer patient recovery ${ }^{[2]}$. Some studies of ROC show large variations $(0.8-2.7 \mu \mathrm{g} / \mathrm{mL})$ in propofol $C_{e}$ producing ROC from anesthesia ${ }^{[3,4]}$, making it difficult to predict the minimum concentration for effective sedation and the concentration of propofol during emergence.

\footnotetext{
* To whom correspondence should be addressed. E-mail hanesth@yuhs.ac

Received 2012-03-01 Accepted 2012-06-03
}

In the absence of individual pharmacodynamic information, propofol is usually dosed on the basis of the average population requirement. Hence pharmacodynamic modeling incorporating a population approach with covariates could be clinically useful for describing the dose-response relationship.

The objectives of our study were: 1) to identify the clinical variables related to the propofol $C_{e}$ at ROC and 2) to apply a population pharmacodynamic modeling approach to data from propofol-remifentanil anesthesia.

\section{Materials and methods}

This study was approved by the ethics committee of the Yonsei University Health System (4-2010-0580). Patients (aged $\geq$ 20 years, ASA I-II) scheduled for elective minor surgery at the Eye and ENT Severance Hospital were included from January 2011 to September 2011. Exclusion criteria were as follows: cardiac, pulmonary, hepatic or renal disease; hearing loss or other neurological deficit; past history of allergy or adverse 
reaction to medication; any type of medication affecting the central nervous system; or body mass index more than 30. All of the patients provided written informed consent.

Anesthesia was induced according to the same standard protocol in all patients. Patients were premedicated iv with $0.1 \mathrm{mg}$ of glycopyrrolate. Anesthesia was induced by effectsite target-controlled infusion (TCI) (Orchestra ${ }^{\circledR}$ Base Primea, Fresenius Vial, France) of propofol and remifentanil after the patients anthropometric data were entered. The pump was operated according to the model for propofol developed by Schnider et $a l^{[5,6]}$ and the model for remifentanil developed by Minto et $a l^{[7,8]}$. The initial target $C_{\mathrm{e}}$ values of propofol and remifentanil were $4 \mu \mathrm{g} / \mathrm{mL}$ and $2 \mathrm{ng} / \mathrm{mL}$, respectively, for induction. Loss of consciousness (LOC) was defined as a patient's inability to open their eyes in response to their name being called loudly, ie, a score of 3 on the Observer's Assessment of Alertness/Sedation Scale $(\mathrm{OAA} / \mathrm{S})^{[9]}$. If LOC was not obtained with this initial $C_{e}$, the $C_{e}$ of propofol was increased in increments of $0.5 \mu \mathrm{g} / \mathrm{mL}$ until LOC occurred. The remifentanil $C_{\mathrm{e}}$ was maintained at $2 \mathrm{ng} / \mathrm{mL}$. Consciousness was assessed every $10 \mathrm{~s}$. At the moment of LOC, the BIS index (BIS VISTA $^{\mathrm{TM}}$, Aspect Medical System, Inc, Norwood, MA, USA) and the $C_{e}$ of propofol and remifentanil were recorded. Rocuronium was given $(0.6 \mathrm{mg} / \mathrm{kg}$ iv) as a neuromuscular blockade. After endotracheal intubation, ventilation was mechanically controlled with $50 \%$ oxygen in an air mixture to maintain the end-tidal carbon dioxide tension at 35 to 40 $\mathrm{mmHg}$. After anesthesia was induced, the $C_{\mathrm{e}}$ of propofol was titrated to maintain BIS values between 40 and 60 throughout the intraoperative period. In addition, the $C_{\mathrm{e}}$ of remifentanil was adapted to intraoperative hemodynamics throughout the surgical procedure.

At the end of surgery, propofol and remifentanil infusion was stopped. The neuromuscular block was antagonized with $0.2 \mathrm{mg}$ of glycopyrrolate and $1.0 \mathrm{mg}$ of neostigmine. Return of consciousness was defined as a score of 3 on the OAA/ S. At the end of surgery and at ROC, the BIS value and the $C_{e}$ of propofol and remifentanil were recorded by an investigator blinded to the conditions. The total amount of propofol and remifentanil, duration of infusion, and the duration of anesthesia and surgery were also recorded. The duration of anesthesia was defined as the time from the start of propofol infusion for induction to extubation of the trachea; the duration of surgery was defined as the time from surgical incision to the application of the last suture. All of the patients were administered ramosetron (Astellas Pharma Inc, Seoul, Korea) $0.3 \mathrm{mg}$ and ketorolac (Hana Pharm Co, Seoul, Korea) $60 \mathrm{mg}$ iv for the prevention of postoperative nausea, vomiting and pain in the operating room before the end of surgery.

Correlations between ROC and several clinical variables were determined by linear correlation analysis. Using the observed ROC, propofol $C_{\mathrm{e}}$ in the basic pharmacodynamic model was distributed between 0 (unconscious) or 1 (conscious). The relationship between the probability of ROC and the propofol $C_{\mathrm{e}}$ was analyzed using a sigmoidal $E_{\max }$ model:

$$
P=1-\frac{C_{\mathrm{e}}^{\lambda}}{C_{\mathrm{e} 50}{ }^{\lambda}+C_{\mathrm{e}}{ }^{\lambda}}
$$

where $P$ is the probability of ROC from anesthesia, $C_{\mathrm{e} 50}$ is the $C_{e}$ associated with $50 \%$ probability of ROC, and $\lambda$ is the steepness of the concentration-versus-response relationship.

The likelihood, $L$, of the observed response, $R$ (unconscious $=0$, conscious $=1$ ) is described by the following equation:

$$
\text { Likelihood }=R \times P+(1-R) \times(1-P),
$$

where $P$ is the probability of ROC.

Model parameters were estimated using the option "LIKELIHOOD LAPLACE METHOD=conditional" in the NONMEM (Nonlinear Mixed Effects Modeling) software (version VII; GloboMax, Hanover, MD, USA). The inter-individual random variability of $C_{\mathrm{e} 50}$ and $\lambda$ was modeled using a log-normal model. For each analysis, NONMEM computes the minimum value of the objective function, a statistic that is proportional to negative twice the log likelihood of the data. To determine the relevant covariates in the final model, a forward inclusion and backward elimination approach was used in consecutive NONMEM runs. A covariate was considered significant when its inclusion lowered the minimum value of the objective function by at least 3.85 points. The difference in the minimum value of the objective function between two nested models was approximately $\chi^{2}$-distributed and could therefore be used for significance tests $(P<0.05$, with one degree of freedom).

\section{Results}

Ninety-four patients met the selection criteria. Fifty-five patients underwent eye surgery and 39 patients underwent ENT surgery. The duration of surgery and anesthesia were $66.9 \pm 53.0$ and $97.5 \pm 54.0 \mathrm{~min}$. The BIS values at baseline and LOC were $92.8 \pm 4.9$ and $67.0 \pm 15.1$. At LOC, the effect-site concentrations $\left(C_{\mathrm{e}} \mathrm{s}\right)$ of propofol and remifentanil were $4.4 \pm 1.1$ $\mu \mathrm{g} / \mathrm{mL}$ and $2.0 \pm 0.3 \mathrm{ng} / \mathrm{mL}$, respectively. At the end of surgery, the BIS value, the $C_{\mathrm{e}} \mathrm{s}$ of propofol and remifentanil were $43.8 \pm 10.6,3.2 \pm 1.0 \mu \mathrm{g} / \mathrm{mL}$ and $2.3 \pm 0.4 \mathrm{ng} / \mathrm{mL}$, respectively. At ROC, the BIS value, the $C_{\mathrm{e}} \mathrm{s}$ of propofol and remifentanil were $75.7 \pm 6.0,1.1 \pm 0.3 \mu \mathrm{g} / \mathrm{mL}$ and $0.8 \pm 1.0 \mathrm{ng} / \mathrm{mL}$, respectively. Table 1 shows the data and correlation coefficients between the propofol $C_{\mathrm{e}}$ at ROC and the analyzed variables. The propofol $C_{e}$ at LOC had a tendency to be positively correlated with the propofol $C_{e}$ at ROC, but this correlation was not statistically significant $(P=0.08)$. With the exception of age, no other clinical variable had a significant correlation with propofol $C_{\mathrm{e}}$ at ROC (Table 1). Age was significantly correlated with the propofol $C_{\mathrm{e}}$ at ROC, with a negative slope $(P<0.01$, Figure 1).

Because age was the only factor that was found to be correlated with propofol $C_{e}$ at ROC, we included this as a covariate in $C_{\mathrm{e} 50}$ and $\lambda$. This pharmacodynamic modeling including age significantly improved the performance of the basic model based on the likelihood ratio test, with a decrease the minimum value of the objective function $(P<0.01)$. Table 2 lists the model parameter estimates for the final selected model. The 
Table 1. Data values and correlation coefficients between several clinical variables and propofol effect-site concentration at return of consciousness. Data are presented as mean \pm SD or number.

\begin{tabular}{|c|c|c|c|}
\hline Clinical variables & Data values & Correlation coefficient & $P$ value \\
\hline Sex (male/female) & $53 / 41$ & 0.03 & 0.76 \\
\hline Age (year) & $42.8 \pm 16.5$ & 0.48 & $<0.0001$ \\
\hline Height (cm) & $167 \pm 10.6$ & 0.11 & 0.28 \\
\hline Weight (kg) & $71.1 \pm 14.4$ & 0.03 & 0.80 \\
\hline Body mass index $\left(\mathrm{kg} / \mathrm{m}^{2}\right)$ & $24.8 \pm 4.4$ & 0.02 & 0.86 \\
\hline Duration of propofol infusion (min) & $79.3 \pm 51.9$ & 0.13 & 0.31 \\
\hline Mean propofol dose during surgery $\left(\mu \mathrm{g} \cdot \mathrm{kg}^{-1} \cdot \mathrm{min}^{-1}\right)$ & $176.3 \pm 97.0$ & 0.12 & 0.24 \\
\hline Mean remifentanil dose during surgery $\left(\mu \mathrm{g} \cdot \mathrm{kg}^{-1} \cdot \mathrm{min}^{-1}\right)$ & $0.1 \pm 0.1$ & 0.16 & 0.13 \\
\hline
\end{tabular}

LOC, loss of consciousness; ROC, return of consciousness.

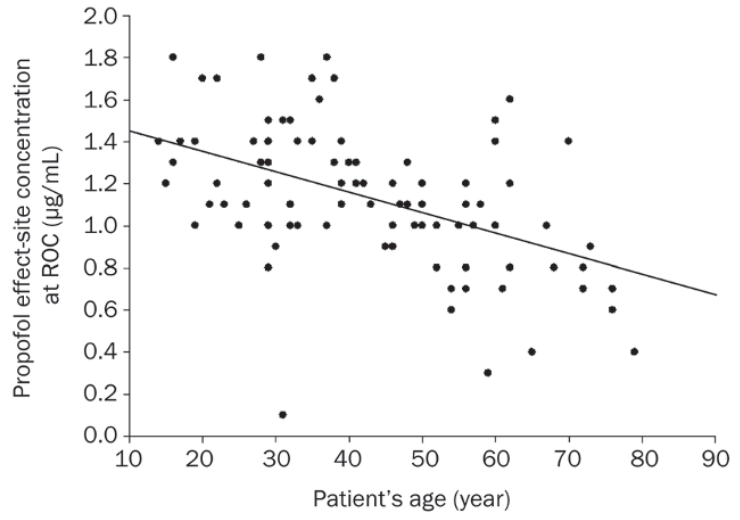

Figure 1. Linear regression between age and propofol effect-site concentration at return of consciousness (ROC). The formula of the regression is $Y=-0.0097 X+1.5472(r=-0.48 ; P<0.01)$.

Table 2. Pharmacodynamic parameters.

\begin{tabular}{|c|c|c|c|c|}
\hline Model & Parameter & Value & $\% \mathrm{CV}$ & OBJF \\
\hline \multirow[t]{2}{*}{ Basic } & $C_{\mathrm{e} 50}(\mu \mathrm{g} / \mathrm{mL})$ & 1.14 & 32.1 & 643.4 \\
\hline & $\lambda$ & 9.03 & - & \\
\hline \multirow[t]{2}{*}{ Final } & $C_{\text {e50 }}(\mu \mathrm{g} / \mathrm{mL})$ & $1.15-0.0128 \times(\mathrm{AGE}-43)$ & 26.0 & 602.6 \\
\hline & $\lambda$ & $9.69-0.141 \times(A G E-43)$ & - & \\
\hline
\end{tabular}

AGE, age in years; CV, coefficient of variation; OBJF, minimum value of objective function; $C_{\mathrm{e} 50}$, effect-site concentration associated with $50 \%$ probability of return of consciousness; $\lambda$, steepness of the concentrationversus-response relationship.

relationship between the probability of ROC and propofol $C_{e}$ is shown in Figure 2. The effect of age on the probability of ROC as evaluated by computed estimation is presented in Figure 3. The values of age used for the predictions correspond to the 25-, 50-, and 75-year-old patients as distributed within the studied population. The $C_{\mathrm{e} 50}$ in $25-, 50-$, and 75 -year-

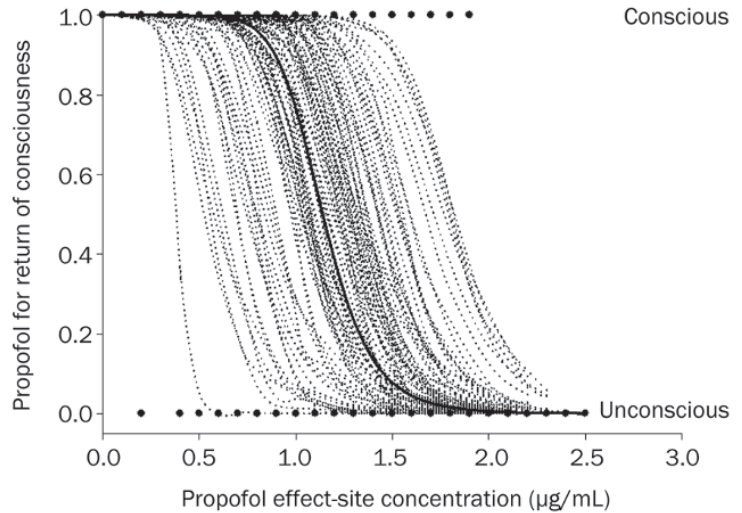

Figure 2. The relationship between the probability of return of consciousness and propofol effect-site concentration. The scattered dots are the raw data observed for all patients. The dotted lines represent individual patient fits, whereas the bold line represents the typical curve of the population data.

old patients was predicted to be $1.38,1.06$, and $0.74 \mu \mathrm{g} / \mathrm{mL}$, respectively. The $\lambda$ in 25-, 50-, and 75-year-old patients was predicted to be $12.23,8.70$, and 5.18 , respectively.

\section{Discussion}

In this study, we searched for clinical factors influencing ROC during emergence from propofol-remifentanil anesthesia and found that age was strongly correlated with ROC. In addition, upon pharmacodynamic modeling, age proved to be a significant covariate of $C_{\mathrm{e} 50}$ and $\lambda$ in the dynamic relationship between propofol $C_{\mathrm{e}}$ and ROC. This study is the first clinical investigation in which pharmacodynamic modeling of ROC has been carried out by incorporating covariates of ROC. It would be clinically advantageous if the individual propofol $C_{e}$ for ROC could be predicted and applied rather than simply targeting a population-based average concentration. A nonlinear dynamic model was chosen to describe the relationship between propofol $C_{e}$ and ROC, as quantal response data 


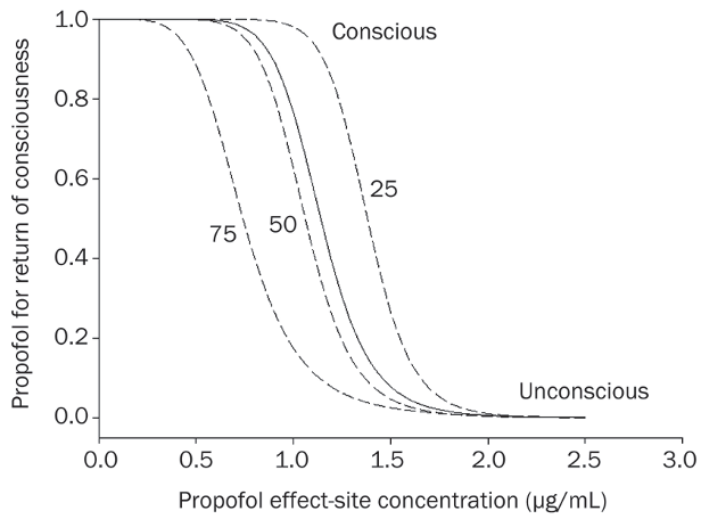

Figure 3. The fit of the logistic regression model is shown. The solid line represents the fit through the data with the age-independent model. The dotted lines are the fits predicted by the age-adjusted regression model for 25-, 50-, and 75-year-old participants.

exhibit a sigmoid relationship. A sigmoidal $E_{\max }$ model using the Hill equation provided a better model than a linear regression since this combination can estimate not only the $C_{\mathrm{e} 50}$ value but the shape or, alternatively, the scale of the concentrationeffect relationship $(\lambda)^{[10]}$. Moreover, the $C_{\mathrm{e} 50}$ values derived from a sigmoidal $E_{\max }$ model are not affected by extreme values to the same extent as in a linear regression model ${ }^{[3]}$.

In the present study, inter-individual variability in propofol $\mathrm{C}_{\mathrm{e}}$ at ROC could be explained by incorporating age as a covariate. Although the patients anthropometric factors except age had little influence on ROC, other potential covariates such as genetics or environmental factors might also play an important role in determining individual ROC and hence contribute to the variability of inter-individual data sets ${ }^{[11]}$. The Schnider pharmacokinetic model was developed based on data from Caucasians, so it may be necessary to examine whether the administration of propofol using a TCI technique based on the Schnider model will provide the same estimated concentration and result in the same dynamic end points in Korean patients.

According to our prediction of the probability of ROC, the propofol $C_{\mathrm{e} 50}$ for a 25-year-old patient is around twice that for a 75-year-old patient. Younger patients may recover consciousness after receiving higher propofol concentrations than could be administered to elderly patients. The $C_{\mathrm{e} 5}$ value, which indicates a $95 \%$ probability that a 25 -year-old patient does not recover consciousness, is around $1.8 \mu \mathrm{g} / \mathrm{mL}$ based on our concentration-response curve. To prevent accidental awareness in young patients with propofol-remifentanil anesthesia, levels at least above this $C_{e}$ should be maintained during surgery. The value of $\lambda$, representing the steepness of the dose-response curve, was greater in younger patients than in older patients. This finding suggests that younger patients may recover more abruptly than older patients, which means that young patients may be easily arousable, able to be extubated, and oriented. However, this may lead to the possibility of trauma as a result of sudden movement, and more attention should be paid to patients safety. The reverse situation could also occur. Elderly patients may experience long and more difficult recovery times. Close monitoring is necessary for elderly patients to prevent re-sedation or respiratory depression due to residual sedative effects during post-anesthetic care after initial ROC.

In older patients, a smaller propofol $C_{\mathrm{e}}$ is required at ROC for both pharmacokinetic and pharmacodynamic reasons. We used the Schnider propofol pharmacokinetic model ${ }^{[5,6]}$, which takes age into consideration, and hence the pharmacokinetic inter-patient variability caused by age would be excluded. In addition, the Schnider model, although still not perfect, has fewer limitations than the other pharmacokinetic models for propofol and therefore has the potential for being the recommended model of choice to be used for $\mathrm{TCI}^{[12]}$. The significant correlation between propofol $C_{\mathrm{e}}$ at ROC and age suggests that age has a considerable influence on the patient's sensitivity to propofol from a pharmacodynamic point of view.

When the effect of remifentanil $C_{\mathrm{e}}$ on ROC was analyzed, we did not find a significant correlation, which is consistent with previous reports ${ }^{[13,14]}$. The $C_{\mathrm{e}}$ of remifentanil $(0.7 \pm 0.8 \mathrm{ng} / \mathrm{mL})$ at ROC was probably too low to affect ROC. However, when predicting the propofol $C_{\mathrm{e}}$ for ROC, it should be kept in mind that the $C_{e}$ values of propofol might be different if another sedative, such as a benzodiazepine or a large dose of remifentanil, is also used. The type of surgery may also influence the propofol $C_{\mathrm{e}}$ at ROC. The $C_{\mathrm{e}}$ of propofol for ROC may be slightly increased in the presence of severe pain caused by major surgeries, eg, thoracic or abdominal surgery, compared to minor surgeries like those in our study ${ }^{[15]}$. The $C_{e}$ of propofol at ROC was not correlated with the duration of propofol infusion or the mean dose of propofol during surgery, which is consistent with a previous study by Kazama et $a l^{[16]}$. These clinical contexts (duration or dose) would influence the time taken to reach an individual's propofol $C_{e}$ for ROC. Current TCI devices display the time required for a calculated $C_{e}$ to decline to a predetermined value of propofol $C_{\mathrm{e}}$ if the infusion is to be stopped. This allows anesthesiologists to predict the time to ROC, provided that the individual $C_{\mathrm{e}}$ at $\mathrm{ROC}$ is known ${ }^{[17]}$.

Although not statistically significant $(P=0.08)$, there was a tendency for the $C_{e}$ of propofol for LOC to positively correlate with the propofol $C_{e}$ at ROC, which suggests that patients requiring a higher propofol $C_{e}$ at LOC tend to recover consciousness at a higher propofol $C_{e}$ as well. We also found that the mean propofol $C_{\mathrm{e}}$ for LOC was higher than that reported in other studies $(4.4 \pm 1.1 \mu \mathrm{g} / \mathrm{mL} \text { vs } 1.25-2.35 \mu \mathrm{g} / \mathrm{mL})^{[6,18]}$. One possible explanation for the higher $C_{e}$ observed in our study is the use of a different pharmacokinetic model. The Schnider model predicts much faster effect-site equilibration with the blood than the Marsh model, as the equilibration constant $\left(K_{\mathrm{eo}}\right)$ is larger in the Schnider model than in the Marsh model (0.459 $\mathrm{min}^{-1}$ vs $\left.0.26 \mathrm{~min}^{-1}\right)$. The predicted $C_{\mathrm{e}}$ in the Schnider model will be higher than that in the Marsh model during the induction period ${ }^{[19]}$.

We note that our study has some limitations. We collected the data of dissipating propofol concentrations after stopping 
infusion, which could lead to a high performance error of TCIbased predictions of propofol $C_{e}$ rather than stable propofol $C_{e}$. To eliminate the confounding effects of pharmacokinetic and pharmacodynamic variability in the response of patients to a certain stimulus, constant $C_{\mathrm{e}}$ values and blood to effectsite equilibration are required ${ }^{[16]}$. However, our study design is more applicable to daily clinical practice during emergence from propofol-remifentanil anesthesia.

We conclude that age significantly affects the pharmacodynamic relationship between propofol $C_{\mathrm{e}}$ and ROC. The propofol $C_{e}$ for ROC can be predicted for individual patients of different ages, and patients can be expected to require shorter recovery times and awaken quickly with early titration of propofol upon surgery completion.

\section{Acknowledgements}

This work was supported in part by the Yonsei University Research Fund of 2009.

The authors thank Dr Go Un ROH MD (Department of Anesthesiology and Pain Medicine and Anesthesia and Pain Research Institute, Yonsei University College of Medicine, Seoul, Korea) for her assistance with analysis of the data.

\section{Author contribution}

Dong-woo HAN and Bon-nyeo KOO designed research; Young-ran KANG and Jae-hoon LEE performed research; Dong-woo HAN, Jeong-rim LEE, Gyu-jeong NOH, and Jaehoon LEE analyzed data; Dong-woo HAN wrote the paper.

\section{References}

1 Larsen B, Seitz A, Larsen R. Recovery of cognitive function after remifentanil-propofol anesthesia: a comparison with desflurane and sevoflurane anesthesia. Anesth Analg 2000; 90: 168-74.

2 Nunes CS, Ferreira DA, Antunes L, Amorim P. Clinical variables related to propofol effect-site concentrations at recovery of consciousness after neurosurgical procedures. J Neurosurg Anesthesiol 2005; 17: $110-4$.

3 Wessén A, Persson PM, Nilsson A, Hartvig P. Concentration-effect relationships of propofol after total intravenous anesthesia. Anesth Analg 1993; 77: 1000-7.

4 Chortkoff BS, Eger El 2nd, Crankshaw DP, Gonsowski CT, Dutton RC, Ionescu P. Concentrations of desflurane and propofol that suppress response to command in humans. Anesth Analg 1995; 81: 737-43.

5 Schnider TW, Minto CF, Gambus PL, Andresen C, Goodale DB, Shafer $\mathrm{SL}$, et al. The influence of method of administration and covariates on the pharmacokinetics of propofol in adult volunteers. Anesthesiology 1998; 88: 1170-82.

6 Schnider TW, Minto CF, Shafer SL, Gambus PL, Andresen C, Goodale
DB, et al. The influence of age on propofol pharmacodynamics. Anesthesiology 1999; 90: 1502-16.

7 Minto CF, Schnider TW, Egan TD, Youngs E, Lemmens HJ, Gambus $\mathrm{PL}$, et al. Influence of age and gender on the pharmacokinetics and pharmacodynamics of remifentanil. I. Model development. Anesthesiology 1997; 86: 10-23.

8 Minto CF, Schnider TW, Shafer SL. Pharmacokinetics and pharmacodynamics of remifentanil. II. Model application. Anesthesiology 1997; 86: 24-33.

9 Chernik DA, Gillings D, Laine H, Hendler J, Silver JM, Davidson $A B$, et al. Validity and reliability of the Observer's Assessment of Alertness/Sedation Scale: study with intravenous midazolam. J Clin Psychopharmacol 1990; 10: 244-51.

10 Lu W, Ramsay JG, Bailey JM. Reliability of pharmacodynamic analysis by logistic regression: mixed-effects modeling. Anesthesiology 2003; 99: 1255-62.

11 Dahaba AA, Zhong T, Lu HS, Bornemann H, Liebmann M, Wilfinger $\mathrm{G}$, et al. Geographic differences in the target-controlled infusion estimated concentration of propofol: bispectral index response curves. Can J Anaesth 2011; 58: 364-70.

12 Masui K, Upton RN, Doufas AG, Coetzee JF, Kazama T, Mortier EP, et al. The performance of compartmental and physiologically based recirculatory pharmacokinetic models for propofol: a comparison using bolus, continuous, and target-controlled infusion data. Anesth Analg 2010; 111: 368-79.

13 Nunes CS, Ferreira DA, Antunes L, Lobo F, Santos IA, Amorim P. Individual effect-site concentrations of propofol at return of consciousness are related to the concentrations at loss of consciousness and age in neurosurgical patients. J Clin Anesth 2009; 21: 3-8.

14 Nho JS, Lee SY, Kang JM, Kim MC, Choi YK, Shin OY, et al. Effects of maintaining a remifentanil infusion on the recovery profiles during emergence from anaesthesia and tracheal extubation. Br J Anaesth 2009; 103: 817-21.

15 Iwakiri H, Nagata O, Matsukawa T, Ozaki M, Sessler DI. Effect-site concentration of propofol for recovery of consciousness is virtually independent of fentanyl effect-site concentration. Anesth Analg 2003; 96: 1651-5.

16 Kazama T, Ikeda K, Morita K, Sanjo Y. Awakening propofol concentration with and without blood-effect site equilibration after short-term and long-term administration of propofol and fentanyl anesthesia. Anesthesiology 1998; 88: 928-34.

17 McCormack J, Mehta D, Peiris K, Dumont G, Fung P, Lim J, et al. The effect of a target controlled infusion of propofol on predictability of recovery from anesthesia in children. Paediatr Anaesth 2010; 20: $56-62$

18 Iwakiri H, Nishihara N, Nagata O, Matsukawa T, Ozaki M, Sessler DI. Individual effect-site concentrations of propofol are similar at loss of consciousness and at awakening. Anesth Analg 2005; 100: 107-10.

19 Barakat AR, Sutcliffe N, Schwab M. Effect site concentration during propofol TCl sedation: a comparison of sedation score with two pharmacokinetic models. Anaesthesia 2007; 62: 661-6. 